\section{Pyrethrum gegen Chloropiden.}

$\mathrm{Zu}$ der Mitteilung: „Massenauftreten von Chloropiden" im Anzeiger für Schädlingskunde, Nr. 4, 1933, S. 59, teilt Herr Dr. R. Menzel, Wädenswil (Schweiz) folgendes mit:

Zur Bekämpfung dieser Fliegen habe ich mit Pyrethrumextrakt gute Erfahrungen gemacht; dieses Mittel übertrifft jedenfalls Flit bei weitem. Auch H. G. H. Kearns: „Early Spring, Swarming of Chloropisca circumdata Meig. with Notes on the Control of the Flies" (Entom. Monthly Mag. 66, 1930) erwähnt Pyrethrumextrakt als am besten wirkend. Ferner sollten beim ersten Erschemen der Fliegen im Spatsommer Fenster usw. mit Gaze überspannt und undichte Stellen verkittet werden. L. Zü rcher, Halmfliegen und Massenansammlungen von solchen (Nitt. Aarg. Nat. Ges. H. 17, 1925) schreibt: „Staubsauger und feinstes Drahtgitter sind vorläufig die einfachsten und wirksamsten Waffen."

\section{Erfolgreiche Bekampfung von Chloropiden mit Pyrethrum. \\ Fon \\ Karl Gïßwald. \\ (Aus dem Institut für angewandte Zoologie, Mïnchen.)}

Ein Notiz von W. Fritsch über das Massenauftreten von Chloropiden (im 19. Jahrgang dieser Zeitschrift, Heft 4) gibt mir Veranlassung zu einer Mitteilung über eine sehr einfache Bekämpfungsweise dieser Getreideschädlinge.

Millionenschwärme von Chloropiden hatten im September 1932 ein Münchener Krankenhaus überfallen und sich hier vor allem an windgeschützten Stellen unter dem Dachvorsprung festgesetzt. Auffallender Weise handelt es sich auch hier, wie in fast allen mitgeteilten Fällen um ein mit wildem Wein bewachsenes Gebäude. Zum Teil waren die Fliegen in Zimmer des obersten Stockes eingedrungen, Decken und Wände waren von ihnen so dicht besetzt, daß die Räume nicht mehr bewohnt werden konnten.

Eine Bestäubung der Fliegen mit dem Pyrethrumkontaktgift Dusturan ${ }^{1}$ ) mittels Handzerstäubungsapparat hatte einen vollen Erfolg. Die von dem Gift getroffenen Fliegen fielen sofort herunter, sammelten sich in einer bis $1 \mathrm{~cm}$ hohen Schicht am Boden; sie zuckten noch schwach und waren in kurzer Zeit tot. Die Zimmer wurden nach der Bestäubung noch eine Stunde geschlossen gehalten, um das Gift besser einwirken zu Jassen. Nach kurzer Durchlüftung konnten dann die Wohnränme wieder bezogen werden. Für den Henschen ist das Dusturan harmlos.

Die Vorliebe der Chloropiden für Gebäude, die mit wildem Wein bewachsen sind, könnte sehr einfach zur Bekämpfung dieser sehr lästigen Getreideschädlinge ausgenuitzt werden durch geejgnete Anlage von weinrebenbewachsenen Fangwänden, die in dem befallenen Gebiet $z u$ errichten wären, möglicherweise transportabel mit eingetopften wilden Reben, so daß man sie je nach Befall und Windrichtung ausstellen kann. Sobald die Fliegen sich hier festgesetzt haben, bietet die Bestäubung mit Pyrethrum keine Schwierigkeiten mehr. Inwieweit sich diese Bekämpfingsmethode durchführen läßt, muß erst noch durch praktische Beobachtung und vor allem durch eingehendere biologische Untersuchung der schädlichen Arten festgestellt werden.

1) Hergestellt von Pflanzenschutzgesellschaft m. b. II. Hamburg.

\title{
Mitteilungen der Deutschen Gesellschaft für angew. Entomologie E.V.
}

Dr. Neiva wurde durch die politischen Wirren gezwungen, S. Paulo für immer zu verlassen. Er wurde in Rio de Janeiro Generaldirektor aller wissenschaftlichen Institute des Ackerbauministeriums. Als solcher hat er das Instituto Biologico Federal gegründet, welche das ehemalige Instituto Biologico des Dr. Moreira und den Jardim Botanico umfaßt, nebst 2 neuen Sektionen
(Genetik, Mikrobiologie). F. Ph. Borgmejer wurde zum Chef der Entom. Abteilung ernannt. Dr. Costa Lima ist Direktor.

\section{Adressenänderung}

Krieg, Dr. H., Altona-Bahrenfeld, Haydnstr. 10. 